\title{
Central Cord Syndrome
}

National Institute of Neurological Disorders and Stroke (NINDS)

\section{Source}

National Institute of Neurological Disorders and Stroke (NINDS). Central Cord Syndrome

Information Page.

Central cord syndrome is the most common form of incomplete spinal cord injury characterized by impairment in the arms and hands and to a lesser extent in the legs. The brain's ability to send and receive signals to and from parts of the body below the site of injury is reduced but not entirely blocked. This syndrome is associated with damage to the large nerve fibers that carry information directly from the cerebral cortex to the spinal cord. These nerves are particularly important for hand and arm function. Symptoms may include paralysis or loss of fine control of movements in the arms and hands, with relatively less impairment of leg movements. Sensory loss below the site of the injury and loss of bladder control may also occur, as well as painful sensations such as tinging, burning, or dull ache. The overall amount and type of functional loss is dependent upon the severity of nerve damage. Central cord syndrome is usually the result of trauma that causes damage to the vertebrae in the neck or herniation of the vertebral discs. It also may develop in persons over the age of 50 due to gradual weakening of the vertebrae and discs, which narrows the spinal column and may contribute to compression of the spinal cord when the neck is hyper-extended. 